\title{
Comunicación
}

\section{Presencia y duración de sonidos cardíacos en caballos sanos en gran altura}

\author{
Presence and duration of cardiac sounds in healthy horses at high altitude
}

Ana María Arévalo, Anamaría Martínez, Laura Sofía Ortíz ${ }^{1}$, Pedro Vargas-Pinto ${ }^{1,2}$

\section{Resumen}

Se da a conocer la presencia y duración de sonidos cardíacos en 24 equinos sanos habitantes de una zona ubicada a $2600 \mathrm{msnm}$. La toma de los datos se realizó mediante fonocardiografía directamente sobre la zona precordial. En todos los equinos se identificaron los sonidos 1 y 2 . El sonido 4 estaba presente en el $87.5 \%$ de los equinos y el sonido 3 fue detectado solo en el $29.2 \%$ de los individuos. El estudio muestra la presencia común del sonido 4 en contraste con la baja presentación del sonido 3 en caballos sanos.

Palabras clave: sonidos cardiacos; fonocardiografía; auscultar; equinos sanos

\section{Abstract}

The presence and duration of cardiac sounds is reported in 24 healthy equines from an area located at 2600 meters above sea level. The data was taken by phonocardiography directly on the precordial area. In all equines, sounds 1 and 2 were identified. Sound 4 was present in $87.5 \%$ of equines and sound 3 was detected only in $29.2 \%$ of individuals. The study shows the common presence of sound 4 in contrast to the low presentation of sound 3 in healthy horses.

Key words: heart sounds; phonocardiography; auscultate; healthy horses

\footnotetext{
${ }^{1}$ Facultad de Ciencias Agropecuarias, Universidad de La Salle, Bogotá, Colombia

2E-mail: pavargas@unisalle.edu.co
}

Recibido: 18 de enero de 2019

Aceptado para publicación: 25 de julio de 2019 


\section{INTRODUCCIÓN}

En el ciclo cardíaco del caballo se generan cuatro sonidos como resultado de una rápida aceleración o desaceleración del flujo sanguíneo y al proceso de llenado ventricular. Dos, tres o cuatro de estos sonidos se pueden escuchar en caballos normales (Marr y Bowen, 2010). Para establecer su presencia se ausculta la zona precordial usando un fonendoscopio electrónico, obteniéndose un registro gráfico de los fenómenos acústicos producidos por el corazón y los grandes vasos sanguíneos (Vargas, 2016).

El primer sonido cardiaco (s1), el cual ocurre al principio de la sístole, es la vibración de las válvulas atrio-ventriculares, cuando estas se cierran y la sangre choca con ellas durante la contracción isovolumétrica. Siempre es detectable y es el que se escucha más fuerte. Examinado desde el electrocardiograma, este sonido ocurre luego del complejo QRS (Palencia Garrido y Lestache, 2003).

El segundo sonido cardiaco (s2) sucede cuando la sangre enviada de retorno hacia los ventrículos choca con las válvulas aórtica y pulmonar cerradas. Se escucha después del s1 con un sonido más corto, pero siempre detectable. El s2 representa el inicio de la diástole. El tercer sonido cardiaco (s3) no se detecta en todos los equinos, y está ligado al s2, lo que hace difícil su diferenciación y es originado por las vibraciones de la pared ventricular durante el llenado de los ventrículos justo después del s2. En forma similar, el cuarto sonido cardiaco (s4) no siempre es detectado, y en la auscultación se escucha al inicio del ciclo cardíaco, antes del s1. Es un sonido principalmente auricular, que corresponde al llenado activo de los ventrículos en la sístole auricular (Marr y Bowen, 2010).

Por lo general, los sonidos cardíacos normales se producen casi simultáneamente en ambos lados del corazón. Estos son detectables mediante la auscultación rutinaria con fonendoscopio. Sin embargo, para su evaluación completa y detallada, se requiere de fonocardiografía. Este estudio buscó establecer la presencia y duración de los sonidos cardiacos en equinos sanos con baja actividad física en gran altura.

\section{Materiales y Métodos}

Se trabajó con 24 equinos mestizos, adultos jóvenes, que se encontraban aparentemente sanos, pertenecientes a dos escuelas de equitación en la Sabana de Bogotá. De estos, 17 fueron machos y 7 fueron hembras de edades diversas, que eran utilizados para práctica de silla y de entrenamiento de exigencia deportiva baja.

Los animales fueron auscultados en reposo para determinar los sonidos cardíacos que podrían ser escuchados en el sitio de trabajo habitual de estos animales. Luego, se hizo la auscultación con un fonocardiógrafo conectado a un Biopac MP35 (BIOPAC Systems, USA) para reconocer y registrar la presencia de los sonidos cardíacos. La auscultación fue realizada en la región precordial izquierda, entre el tercer y cuarto espacio intercostal al nivel de la base del corazón. La captura y proceso de los datos se realizó mediante el software AcqKnowledge ${ }^{\circledR}$.

La duración de los sonidos fue medida desde la primera separación de la línea base hasta el punto donde las ondas vuelven a incorporarse a la línea base. Esta medición se realizó tres veces por individuo por cada sonido. Los valores obtenidos para cada sonido fueron promediados por individuo. Los datos son presentados mediante estadística descriptiva. Adicionalmente, las duraciones de s1 y s2 fueron comparados mediante la prueba de t-Student. La presencia de cada sonido fue presentada como porcentaje. El software utilizado fue Graphpad Prism ${ }^{\circledR}$. 


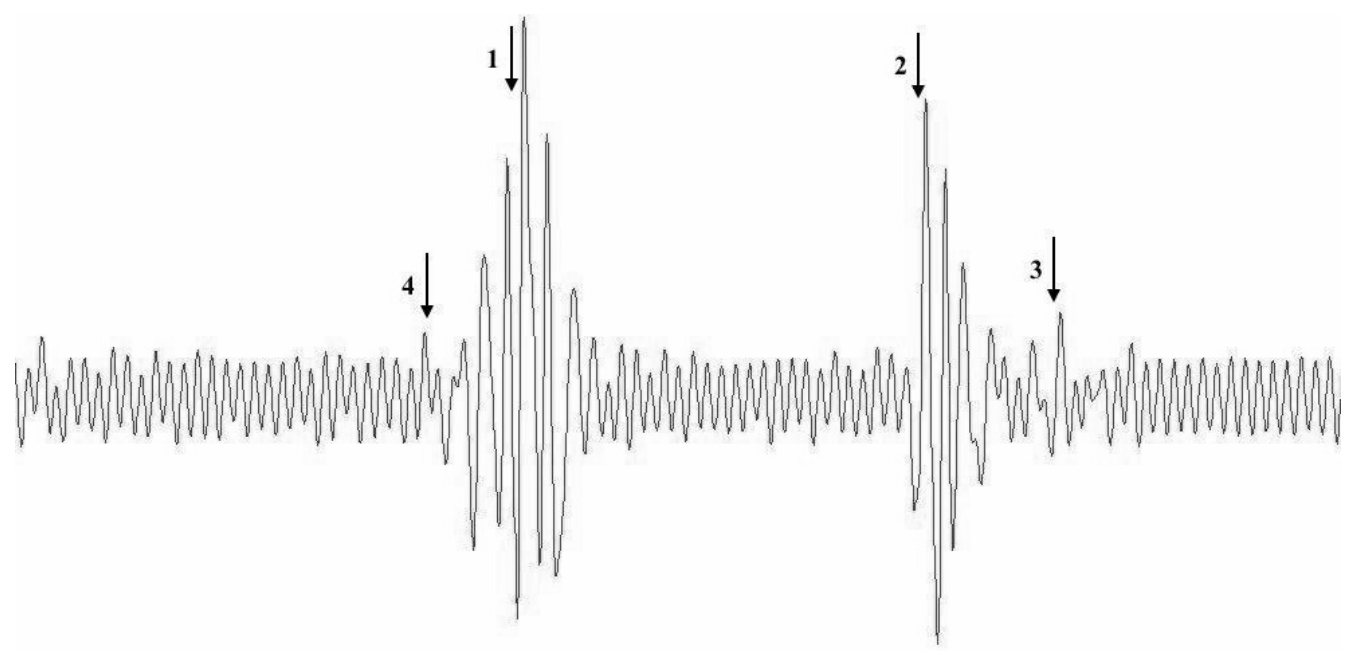

Figura 1. Fonocardiograma obtenido en un caballo adulto sano. Nótese la presencia de sonidos s4 (4), s1 (1), s2 (2) y s3 (3)

Duración de Sonidos Cardiacos

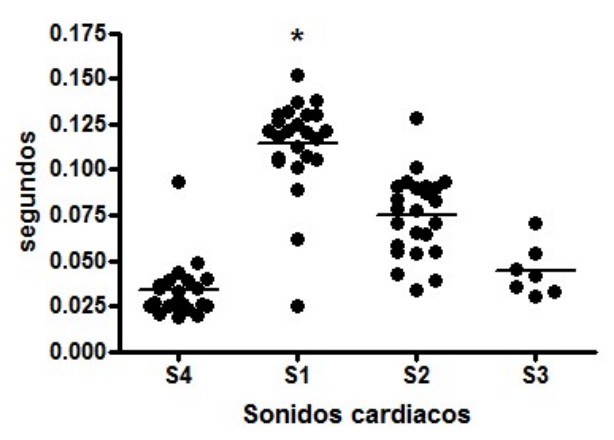

Figura 2. Duración de los sonidos cardíacos en caballos. El s1 es significativamente más largo que los demás sonidos presentes.

\section{Resultados}

En el fonocardiograma de la Figura 1 se puede observar el s4 al inicio (4), con una menor amplitud y duración que el s1 (1) que se caracteriza por una serie de ondas de gran amplitud. Le sigue un espacio de tiempo que precede al s2 (2); también de gran amplitud, pero menor que del s1 y con una menor duración. Por último, el s3 (3) que se observa al final separado del S2 y con una ampli-

\section{Porcentajes s3 y s4}

$\begin{array}{r}100,00 \% \\ \hline 90,00 \% \\ \hline 80,00 \% \\ \hline 70,00 \% \\ 60,00 \% \\ \hline 50,00 \% \\ 40,00 \% \\ \hline 30,00 \% \\ \hline 20,00 \% \\ \hline 10,00 \% \\ \hline 0,00 \% \\ \hline\end{array}$

Figura 3. Porcentajes de caballos que presen taron sonidos cardiacos s 3 y s 4

tud de onda y duración menor. En ninguno de los animales examinados se detectaron soplos o algún otro sonido adicional.

La Figura 2 muestra el promedio de la duración de los sonidos cardíacos, siendo el s1 el de mayor duración. En la Figura 3 se muestran los porcentajes de los individuos que presentaron s3 y s4. El 29.2\% (7) de los individuos presentaron s3 y el $87.5 \%$ (21) presentaron s4. 


\section{Discusión}

Los sonidos cardiacos encontrados mediante fonocardiografía corresponden a los reportados en la literatura en cardiología equina (Klein, 2014). Sin embargo, cabe resaltar que no existe claridad sobre los porcentajes de animales que presentan los sonidos s3y s4. Si bien es sabido que estos son sonidos normales y se conoce su fisiología, es muy importante conocer que no todos los animales lo presentan y que, como se vio en este estudio, es mucho más común la presencia de s4 que la de s3. El s3 no es fácilmente detectable debido a su proximidad al s2; sin embargo, el uso de la fonocardiografía permite establecer de una manera más objetiva su presencia y representa una ayuda para el clínico de campo.

La literatura reconoce que el s3 es un sonido cardiaco fisiológico en caballos y está presente en todas las razas (Boffi, 2007), pero solo se encontró en el $29.2 \%$ de los animales, tanto por auscultación como por fonocardiografía. Este bajo porcentaje pudo ser debido a algún grado de distorsión del trazado generado por ruido medioambiental, el cual solaparía al s3, así como por su baja ampli- tud. Sin embargo, la auscultación y el fonocardiograma llevaron a los mismos resultados en la búsqueda del s3 en este grupo de animales.

Por otro lado, el s4 ocurre antes que cualquier otro sonido cardiaco del ciclo y, por esto, es fácilmente auscultable e identificable en el fonocardiograma, estando presente en el $87.5 \%$ de los individuos muestreados. Los datos generados por auscultación y por fonocardiografía fueron los mismos.

\section{Literatura Citada}

1. Boffi F. 2007. Fisiología del ejercicio en equinos. Buenos Aires, Argentina: Intermedica. $320 \mathrm{p}$.

2. Klein B. 2014. Fisiología veterinaria de Cunningham. Barcelona, España: Elsevier. $607 \mathrm{p}$.

3. Marr-Bowen I. 2010. Cardiology of the horse. $2^{\text {nd }}$ ed. Edinburgh: Elsevier. 300 p.

4. Garrido-Lestache P. 2003. Cardiología equina. Métodos de exploración cardiaca (I). Profesión Vet 15: 30-37.

5. Vargas Amaral F. Monte A. 2016. Fonocardiograma: construcción y evaluación. Educación Física y Ciencia 18(1): 1-8. 